\title{
shRNA libraries and their use in cancer genetics
}

\author{
René Bernards $^{1}$, Thijn R Brummelkamp ${ }^{1,2}$ \& Roderick L Beijersbergen ${ }^{1}$ \\ RNA interference was originally described as a powerful tool to inhibit gene expression \\ in model organisms. Until recently, loss-of-function genetic screens in mammalian \\ cells were hampered by a lack of suitable tools that can be used in a high-throughput \\ format. Here we discuss the construction of short-hairpin RNA (shRNA) vector libraries, \\ in particular those generated at the Netherlands Cancer Institute (NKI), and their \\ application in mammalian cancer genetics. We describe their virtues and limitations, as \\ well as different options for screening such libraries.
}

The discovery five years ago that short interfering RNAs (siRNAs) can also silence gene expression in higher organisms has revolutionized loss-of-function genetic screens in mammalian cells ${ }^{1}$. The use of such chemically synthesized duplex RNAs has several advantages, including the constant quality of reagents (important in high-throughput approaches), the ability to chemically modify the RNA molecules (improving stability and delivery) and the possibility of controlling the amount of silencing reagent delivered to the cell. Disadvantages of using siRNA silencing reagents include the fact that siRNAs are short-lived, which results in the transient inhibition of gene expression only, and the difficulty of efficiently delivering siRNAs to (nondividing) primary cells. Furthermore, the cost of siRNAs also becomes an issue, especially in genome-wide high-throughput approaches.

To deal with these limitations, we and others have developed vectors that produce shRNAs, which are processed intracellularly into short duplex RNAs having siRNA-like properties ${ }^{2,3}$. Such vectors provide a renewable source of a gene-silencing reagent that can mediate persistent gene silencing after stable integration of the vector into the host-cell genome. Furthermore, the core silencing 'hairpin' cassette can be readily inserted into retroviral, lentiviral or adenoviral vectors, facilitating delivery of shRNAs into a broad range of cell types ${ }^{4-8}$. Additonally, regulatable versions of shRNA vectors have been generated and used successfully in genetic screens ${ }^{9,10}$.

\section{The NKI shRNA library collections}

shRNA expression cassette. The NKI shRNA library is based on the production of a shRNA expressed from a vector harbouring the RNA polymerase III-driven promoter for H1 RNA, the RNA component of human RNase P. This shRNA consists of a target transcriptspecific 19-base double-stranded stem, connected by a 9-base loop sequence ${ }^{2}$ (Fig. 1). Upon expression in cells, this hairpin RNA is processed into a functional siRNA consisting of a 19-base-pair dsRNA with 2-nucleotide single-stranded overhangs on each end, which is virtually identical to the synthetic siRNAs that initially had proven to be very efficient in gene silencing in mammalian cells ${ }^{1}$. At present, the NKI shRNA library targets $\sim 8,000$ human genes and $\sim 15,000$ mouse genes, with 3 or 2 different shRNA constructs per gene, respectively. The features of the libraries are summarized in Box $\mathbf{1}$.

Vector backbone. The retroviral vector used for the library construction is based on a mouse stem cell virus (MSCV) self-inactivating retroviral backbone. The $\mathrm{H} 1$ promoter is cloned in the opposite orientation to the PGK promoter, which directs the expression of a

${ }^{1}$ Division of Molecular Carcinogenesis and Centre for Biomedical Genetics, The Netherlands Cancer Institute, Plesmanlaan 121,1066 CX Amsterdam, The Netherlands. ${ }^{2}$ Present address: Whitehead Institute, 9 Cambridge Center, Cambridge, Massachusetts 02142, USA. Correspondence and requests for materials should be addressed to R.B. (r.bernards@nki.nl) or R.L.B. (r.beijersbergen@nki.nl).

PUBLISHED ONLINE 23 AUGUST 2006; DOI:10.1038/NMETH921 
puromycin selectable marker ${ }^{4}$. The puromycin marker can be used for rapid selection of infected cells. Owing to problems of recombination observed with the original pRETRO-SUPER vector under certain (adverse) culture conditions, we included the chloramphenicol bacterial selectable marker in close proximity to the hairpin cassette, yielding pRSC (Fig. 1). This marker serves to prevent recombination of the vector during low-volume bacterial culturing used for high-throughput plasmid DNA isolation and replication of glycerol stocks. pRSC plasmids, which are subjected to multiple rounds of replication in adverse bacterial culture conditions indeed remain stable and do not show any signs of recombination when cultured in chloramphenicol-containing media (unpublished data).

Selection of shRNA sequences. For the human shRNA library, three target transcript-specific 19-mer sequences were selected using selection criteria described previously ${ }^{11}$. For the more recent mouse shRNA vector library, two shRNA vectors were generated per transcript. In brief, the sequences were selected so that they do not contain stretches of four or more thymine or adenine residues, to have a $30-70 \% \mathrm{G}+\mathrm{C}$ content, to target the coding sequence of an mRNA, to begin with a guanine or cytosine residue, not to contain EcoRI or XhoI restriction sites, and to share minimal sequence similarity to other genes (see ref. 11 for more details on design rules). For the more recent mouse shRNA library, we also followed the thermodynamic asymmetry rule in the 19-mer design to optimize recruitment of the correct RNA strand into the RNA-induced silencing complex (RISC) ${ }^{8,12}$.

Library construction. Based on the 19-mer sequence, two complementary 60-mer oligonucleotides were generated comprising the stem region as defined by the 19-mer target sequence, separated by a 9-base loop sequence. The 60-mers were designed such that they have overhangs compatible with BglII and HindIII sites after annealing, allowing rapid cloning into pRSC (Fig. 1). The expression cassette compromising the $\mathrm{H} 1$ promoter and the hairpin construct can be shuttled into a different backbone by EcoRI-XhoI digestion (the absence of these two restriction sites was an additional 19-mer design rule). The application of the bar-code screening technology (see below) is compatible with shuttling of the shRNA library to different backbones such as lentiviral or adenoviral vectors in a polyclonal fashion. Presently, we have recloned both the human and mouse shRNA vector collections into a lentiviral backbone (using the vector described in ref. 5; unpublished data). Additionally, the shRNA cassette can be transferred to a retroviral backbone harboring a yellow fluorescent protein (YFP) expression cassette to perform competitive analysis of selective growth advantage or disadvantage caused by specific-gene inactivation ${ }^{13,14}$.

\section{Library introduction into target cells}

The pRSC vector can be introduced into the target cells either by transfection or retroviral infection. The introduction of
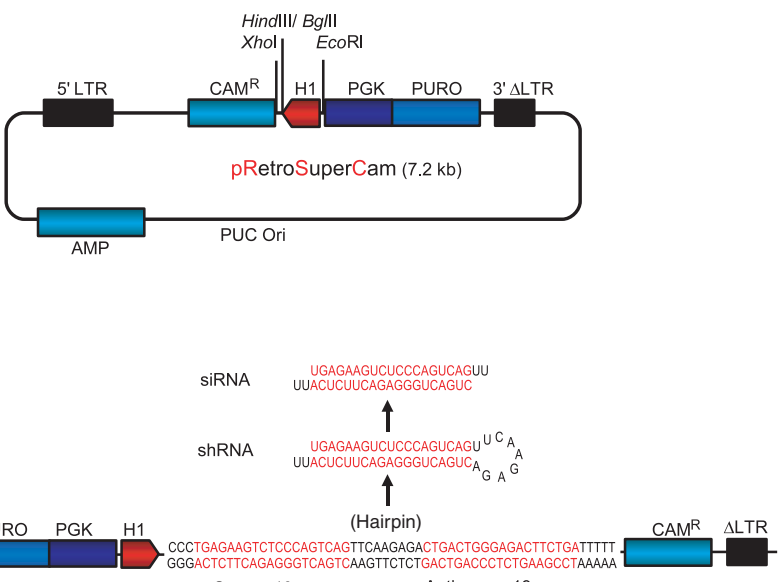

Sense, 19-mer

Antisense, 19-mer

Figure 1 | Construction of the NKI shRNA library. The pRSC vector contains an H1 promoter in a selfinactivating retroviral backbone including two bacterial resistance markers (chloramphenicol and ampicillin) and a puromycin-selectable marker for mammalian cells. The shRNA inserts are cloned as shuttling into a different vector backbone (lentiviral or adenoviral). LTR indicates the retroviral long terminal repeat; $\Delta \mathrm{LTR}$, this repeat with the self-inactivating deletion.

shRNA vectors by transient transfection allows for analysis of short-term phenotypes, whereas infection can be used both in short- and long-term experiments. Virus can be produced using standard procedures and packaging systems to generate retroviral supernatants, and depending on the need and safety issues, these can be ecotropic (and introduced in rodent cells or human cells modified to express the ecotropic receptor) or pantropic. If high viral titers are necessary for transduction of the library, concentrated viral supernatants can be generated. The MSCV-based vector allows expression in stem cells and embryonic stem cells as it avoids silencing by methylation. We have yet to find a (dividing) cell type in which the shRNA cassette cannot be efficiently expressed using the MSCV vector backbone.

\section{Barcoding technology}

We have previously described a technology to expedite the screening of complex shRNA libraries, which we named shRNA barcode screening ${ }^{11,15,16}$. Briefly, the technology takes advantage of the fact that each knockdown vector that is stably integrated into the host cell genome carries a unique 19-mer molecular 'bar code' that can serve to identify the gene that is knocked down in the bar-coded cell (Fig. 2). In our system, the 19-mer of the shRNA vector itself is used as a molecular bar code, as this sequence is unique and has a $\mathrm{G}+\mathrm{C}$ content of between 30 and $70 \%$, making the hybridization temperatures of the bar code-containing oligonucleotides similar. Furthermore, the shRNA vector design criteria did not allow a greater than a 15 out of 19 match with another 19-mer in the library, which also assures specificity of hybridization.

Others have used an 'external' bar code, in which a separate random 60-mer oligonucleotide is linked in cis to the shRNA vec$\operatorname{tor}^{17,18}$. Note that the oligonucleotides that we spot on the microarray for bar-code hybridizations are not the 19-mer sequences, but the 60-mer oligonucleotides that were used to generate the shRNA library (see Fig. 1). The use of the longer oligonucleotides 
represents a substantial cost savings in this approach, as only small amounts of 60-mer oligonucleotide are required to clone the shRNA library vectors. As a result, substantial amounts of oligonucleotide are available for spotting of bar-code microarrays. We have demonstrated that the use of shRNA vector oligonucleotides for microarray hybridization generates specific signals with high intensity and can be used in a quantitative manner to measure the relative abundance of each individual shRNA vector in a complex population of cells infected with the entire NKI human shRNA library of some 24,000 vectors ${ }^{19}$.

The bar-code sequences are recovered from cell populations by PCR amplification, and therefore it is important that all the different hairpins can be amplified efficiently. Optimization of primer design and PCR conditions allows the efficient amplification of all hairpin sequences from genomic DNA. Using our PCR protocols we have not observed difficulties in PCR amplification of specific hairpin sequences and were able to amplify the bar codes in a complex mixture without losing complexity of the bar-code mixture. Using this method, we can identify differences in relative abundance of bar codes of fourfold up to more than 64-fold in a linear fashion ${ }^{19}$.

The bar-code technology can be applied to a wide range of biological questions, the only criterion being that the biological effect either cause a selectable phenotype based on cell survival or proliferation, or cause cellular phenotypes that can be identified based on selectable features such as marker expression, adhesion or migration (reviewed in refs. 15,20). The bar-code technology is in principle suited for both positive and negative selection. Examples of positive-selection bar-code screens show that this technique can be applied to identify drug targets, to gain insight into the mechanism of drug action and to identify genes that cause resistance to a specific drug ${ }^{16,21}$. Although the cell population exposed to the drug needs to show an increase in the relative amount of shRNA cassettes of at least fourfold to be identified as a potential hit, this can usually be accomplished by simply increasing the duration of of the cell-population selection in the presence of the drug. As a result, even vectors that give a relatively weak phenotype (for instance because of partial knockdown of the target) are often enriched in a typical bar-code screen for drug resistance. From various experiments using the human shRNA library that has three hairpin vectors per gene, we estimate that for about one-third of the hits, enrichment of two or more hairpin vectors is observed in a bar-code screen.

The application of this technology for negative selection (that is, identification of shRNA vectors that cause either growth arrest or cell death) is more challenging. To identify loss of shRNA vectors from a population, one needs a system in which (adapted from ref. 16). the specific shRNA vector causes a phenotype, which is sufficiently penetrant to score for the relative depletion of cells carrying the shRNA vector from the population. At present, there is only a single example of a polyclonal shRNA screen in mammalian cells that identified shRNAs causing a dramatic reduction in cell viability ${ }^{9}$. Presently single-well arrayed format screens are favored for negative selection screens with shRNA libraries (see below).

\section{Screening strategies}

The large collections of shRNA vectors can be used in many different ways to identify genes associated with specific cellular phenotypes. A list of published screens performed with our shRNA libraries is presented in Table 1. One approach is to select a small set of shRNA vectors that target a specific gene family or a subset of vectors comprising components of a molecular pathway of interest. The relatively small scale allows screening for relatively labor-intensive phenotypes. For instance, we have identified the deubiquitinating (DUB) enzyme responsible for removing ubiquitin from the Fanconi Anemia D2 protein in a shRNA screen using 55 DUB knockdown vector sets in a western blot format ${ }^{22}$.

Additionally, genome-wide collections of shRNA vectors allow performance of large-scale screens, for which different screening formats can be used. The major distinction between screening formats
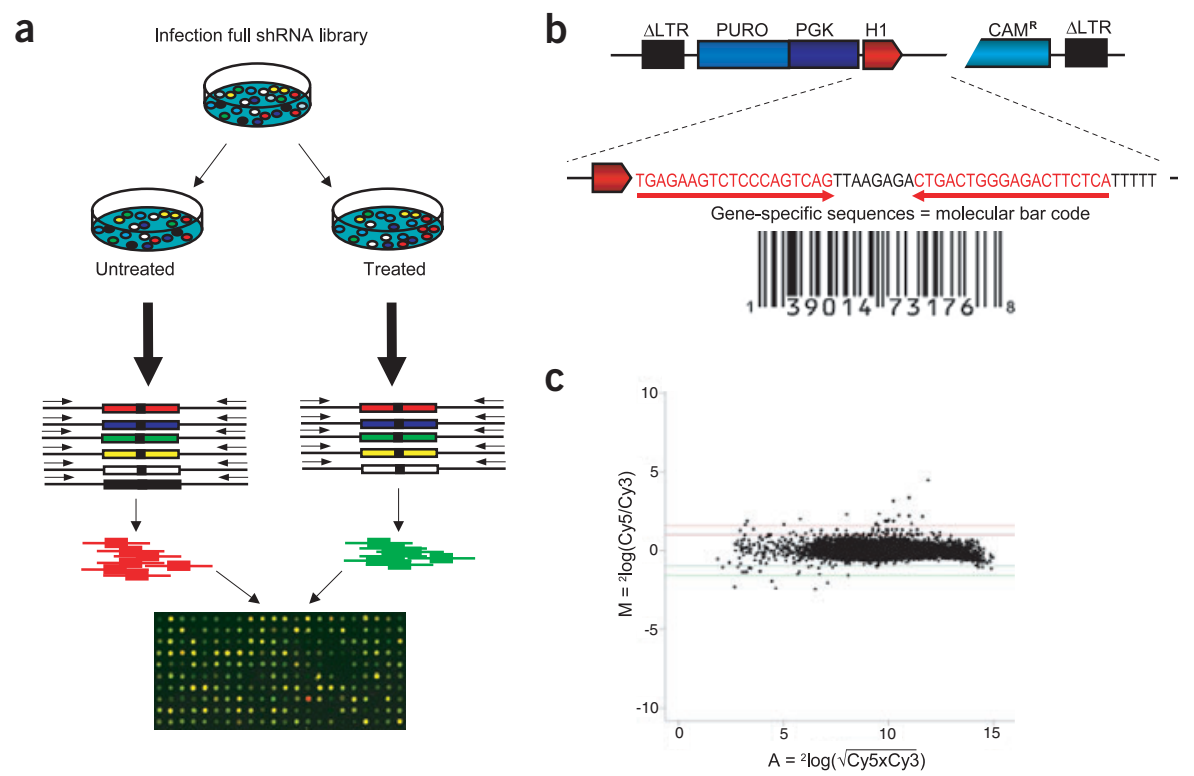

Figure 2 | siRNA bar-code screens. (a) Schematic outline of the bar-code technology. A large population of cells is infected with the NKI shRNA library and divided into two populations. One is treated (or selected), whereas the other population serves as a reference for the hybridization. From each population, genomic DNA is isolated and shRNA cassettes are recovered by PCR. The PCR products are used as templates for an in vitro linear amplification reaction to generate RNA probes that are subsequently labeled with fluorescent dyes. The probes from the selected cells and reference population are hybridized to a DNA microarray containing the complementary sequences from the complete NKI shRNA library. (b) An integrated shRNA vector also delivers a gene-specific identifier in the form of a unique 19-mer 'molecular bar code' that can be used to identify which cell carries any given knockdown allele in a large population of cells. (c) An example of the result of a bar-code hybridization. The graph depicts the $\log _{2}$ of the average intensity versus the $\log _{2}$ of the fluorescence intensity ratio. shRNA vectors that are enriched by the selection method are represented by spots with an increased ratio 
is single well-based arrayed format screens versus 'polyclonal' screens, in which the entire library of shRNA vectors is infected in cells in a pooled format. Single well-based screens make it possible to identify shRNA vectors, which induce phenotypes that can only be measured in well-based or even individual cell-based readouts. The use of wellbased screens, however, is mostly limited to short-term readouts because it is practically impossible to passage cells in arrayed assays, whereas polyclonal screens can be used to select for long-term phenotypes.

The NKI shRNA library has been developed with particular emphasis on polyclonal screens. In this format, the complete set of shRNA vectors is pooled, and this collection is introduced into a large population of cells by viral infection. This population of cells is then subjected to a selective condition, such as induction of a growth arrest, exposure to a cytostatic drug or induction of apoptosis. After this selection, surviving and proliferating colonies can be recovered and the shRNA inserts can be identified by PCR amplification, cloning and sequence identification. This method has been very successful in the isolation of several genes that encode proteins in cancer-relevant pathways such as the p53 pathway and RAS-dependent transformation ${ }^{11,23}$.

One requirement for this type of screen is a low background of spontaneously occuring resistant cells in the cell system used and that a substantial growth advantage is gained by the cells harboring the shRNA vector. A screening system that is usable for such approaches usually shows a background of spontaneously resistant cells of less than 1 in 10,000 cells. If these requirements are not met, the bar-code screening technology can often circumvent such technical problems. Bar-code screening relies on the selective enrichment of certain shRNA vectors in a large population of shRNA vector-infected cells. As a result, background colonies will have a random distribution of all shRNA vectors in the population. In contrast, active shRNA vectors will be enriched to a much higher degree because of their specific effect on cellular phenotype under the conditions of the screen. As a consequence, the relative increase in abundance of active shRNAs will be much greater than those present in background colonies. Furthermore, the measurement of the relative abundance of shRNA vectors by microarray hybridization is very sensitive. A small increase in relative abundance can be determined with high significance, and its reproducibility can readily be tested in independent biological samples of the same type of screen.

Finally, similar to single-well screens in which multiple independent siRNA sequences targeting a single gene are used in separate wells, the bar-code system also facilitates the identification of multiple independent shRNA vectors that target the same gene and are enriched in the screening system, thereby reducing the chance of an off-target effect of any particular shRNA.

\section{Outlook}

To facilitate genome-wide screens using RNAi, several groups have developed libraries of shRNA-producing vectors. The major characteristics of the shRNA library that we have developed at the NKI are summarized in Box $\mathbf{1}$.

An important new concept in cancer-drug discovery, first presented in 1997 (ref. 24), is that of genotype-specific drugs: drugs that only act in a cancer-specific genetic context. Such cancerspecific drug targets are much sought after, as their inhibition should in theory be far more toxic to cancer cells than to normal cells. In principle, large-scale loss-of-function genetic screens in mammalian cells are very well suited for the identification of genes, whose suppression is lethal only in the context of a cancerspecific genetic alteration. A complicating factor in such screens is that one has to select for vectors that are lost from a population of cells rather than enriched, which is technically more challenging. But there are ways around this problem. For instance, one could positively select for cells that enter into apoptosis by selecting cells (using, for example, fluorescence-activated cell sorting) that stain positively for apoptotic markers. By recovering the shRNA vectors from apoptotic cells, one can recover the (very small) bar-code cassette by PCR and quantify the relative abundance of all shRNA vectors in the apoptotic population in a bar-code experiment. We have recently shown that the length of the DNA in apoptotic cells is sufficient to recover bar-code sequences by PCR (data not shown).

A second appealing approach will be the implementation of socalled 'high-content' screening technology into shRNA screens ${ }^{25}$. High-content screening technology allows the identification of complex cellular phenotypes through automated (confocal) microscopic analysis of individual cells in high-throughput format. By combining this technology with single-well transfections or infections with shRNA vector sets, one can score for a range of phenotypes in a variety of cellular pathways.

For certain cancers, translation into drug development of our molecular understanding of the genetic alterations underlying the disease has already resulted in dramatically improved therapy. For instance, Imatinib (Gleevec) in chronic myeloid leukemia and gastrointestinal stromal tumors, and Trastuzumab (Herceptin) in HER2-positive breast cancer are examples of 'targeted' therapeutics, which are effective and selective because they target 


\section{BOX 1 NKI LIBRARY AT A GLANCE}

\section{Library vector}

Vector description. Library vector: pRSC (see Fig. 1; ref. 4). Viral vector backbone: self-inactivating retroviral vector MSCVSIN. Plasmid derivative of pRETRO-SUPER.

Major modification: addition of chloramphenicol resistance cassette for selection to improve vector stability in bacteria. Additional features: shRNA expression cassette under the control of H1 pol III promoter, a PGK-puromycin marker and ampicillinresistance marker for selection.

Library stability. Recombination may occur when the bacteria are cultured under suboptimal growth conditions or with repeated freeze-thaw cycles. This is suppressed by chloramphenicol selection.

Infectivity. The libraries can be used to infect dividing cells. Titers and infectivity are mostly dependent on the packaging systems that are used. This vector is compatible with all commonly used packaging systems for retroviruses.

The library has been successfully introduced into a large variety of different cancer cell lines and primary cells. Expression of the hairpin RNAs also works in undifferentiated (embryonic stem) cells.

Silencing. Silencing may occur depending on the integration site. The MSCV-based backbone is relatively resistant to chromatin-mediated silencing.

Stability of knockdown phenotype has been observed for several months in the cell systems used so far.

\section{Library production}

Vector DNA production yield. The yield strongly depends on the method of DNA isolation. This is a high-copy-number plasmid that can be isolated and purified from bacteria using standard protocols.

Viral titer. Production conditions: virus-containing supernatants are produced using packaging cells transiently transfected with pRSC-shRNA vectors. Depending on the type of packaging system different viral subtypes can be produced (retroviral, lentiviral or adenoviral).

Titer depends on packaging system.

Virus titer estimated by selection for puromycin-resistant cells or detection of the shRNA expression cassettes in target cells by PCR amplification and sequencing, or bar-code array hybridization is typically $>10^{6}$ I.U./ml.

\section{Library coverage}

As of July 2006, the library contains shRNA directed against: $\sim 8,000$ human genes $(24,000$ shRNAs)

$\sim 15,000$ mouse genes $(\sim 30,000$ shRNAs)

Gene coverage distribution. $~ 3$ shRNAs per human target gene; $\sim 2$ shRNAs per mouse target gene.

Region of transcripts targeted: $~ 100 \%$ open reading frames (ORFs).

List of target genes: see supplementary information in reference 11 and http://www.screeninc.nl/gene/index.php.

shRNA sequences. The design rules for the human shRNA library have been published ${ }^{11}$. For the mouse library, the thermodynamic asymmetry rules ${ }^{8,12}$ were also taken into consideration in the target sequence design ${ }^{11}$.

List of shRNA sequences: not available.

\section{Functional tests}

Sequence verification. The available collection of single vectors is verified by sequencing of one strand.

Validation data. 288 vectors targeting 96 human genes were tested for knockdown of ORF product- $\beta$-galactosidase fusion proteins.

Knockdown efficiency. Of the tested shRNAs, $30 \%$ give more than $70 \%$ knockdown (as single vectors).

For $70 \%$ of tested target genes there is $>70 \%$ knockdown when tested with a pool of three shRNA vectors.

For $70 \%$ of tested target genes, the library contains at least 1 shRNA that provides $>70 \%$ knockdown efficency.

For $10 \%$ of tested target genes, the library contains at least 2 shRNAs that provide $>70 \%$ knockdown efficiency.

Nonspecific off-target effect. Off-target effects cannot be excluded, and extensive follow up on hits from a screen is needed. Multiple hairpin vectors that show the same phenotype can decrease the risk of off-target effects certainly when the phenotype is very specific.

\section{Distribution}

Sequence-verified versions of the libraries will be available from Geneservice Ltd.

The libraries will be available as glycerol stocks of single vectors. Sub-collections (targeting certain pathways or classes of genes) will be available. cancer-specific genetic alterations. But the number of genes that are consistently either mutated or over-expressed in cancer is limited, hampering future development of novel targeted therapeutics. Through functional genetic approaches, such as those described above, it will be possible to uncover a completely new arsenal of powerful cancer-selective drug targets. In the longer term this should lead to the identification of completely new classes of drugs that exploit the vulnerabilities of the cancer cell that result from their genetic deficiencies.

\section{ACKNOWLEDGMENTS}

We thank A. Fabius and A. Dirac for critical reading of the manuscript. This work was supported by grants from the Netherlands Genomics Initiative, The EU 6th framework integrated project "INTACT", the centre for Biomedical genetics (CBG) and the Dutch Cancer Society (KWF). 


\section{COMPETING INTERESTS STATEMENT}

The authors declare competing financial interests (see the Nature Methods website for details).

\section{Published online at http://www.nature.com/naturemethods/} Reprints and permissions information is available online at http://npg. nature.com/reprintsandpermissions/

1. Elbashir, S.M. et al. Duplexes of 21-nucleotide RNAs mediate RNA interference in cultured mammalian cells. Nature 411, 494-498 (2001).

2. Brummelkamp, T.R., Bernards, R. \& Agami, R. A system for stable expression of short interfering RNAs in mammalian cells. Science 296, 550-553 (2002).

3. Paddison, P.J., Caudy, A.A., Bernstein, E., Hannon, G.J. \& Conklin, D.S. Short hairpin RNAs (shRNAs) induce sequence-specific silencing in mammalian cells. Genes Dev. 16, 948-958 (2002).

4. Brummelkamp, T.R., Bernards, R. \& Agami, R. Stable suppression of tumorigenicity by virus-mediated RNA interference. Cancer Cell 2, 243-247 (2002).

5. Dirac, A.M. \& Bernards, R. Reversal of senescence in mouse fibroblasts through lentiviral suppression of p53. J. Biol. Chem. 278, 11731-11734 (2003).

6. Michiels, F. et al. Arrayed adenoviral expression libraries for functional screening. Nat. Biotechnol. 20, 1154-1157 (2002).

7. Stegmeier, F., Hu, G., Rickles, R.J., Hannon, G.J. \& Elledge, S.J. A lentiviral microRNA-based system for single-copy polymerase II-regulated RNA interference in mammalian cells. Proc. Natl. Acad. Sci. USA 102, 13212-13217 (2005).

8. Khvorova, A., Reynolds, A. \& Jayasena, S.D. Functional siRNAs and miRNAs exhibit strand bias. Cell 115, 209-216 (2003).

9. Ngo, V.N. et al. A loss-of-function RNA interference screen for molecular targets in cancer. Nature 441, 106-110 (2006).

10. Van De Wetering, M. et al. Specific inhibition of gene expression using a stably integrated, inducible small-interfering-RNA vector. EMBO Rep. 4, 609-615 (2003).

11. Berns, K. et al. A large-scale RNAi screen in human cells identifies new components of the p53 pathway. Nature 428, 431-437 (2004).

12. Schwarz, D.S. et al. Asymmetry in the assembly of the RNAi enzyme complex.
Cell 115, 199-208 (2003).

13. Torrance, C.J., Agrawal, V., Vogelstein, B. \& Kinzler, K.W. Use of isogenic human cancer cells for high-throughput screening and drug discovery. Nat. Biotechnol. 19, 940-945 (2001).

14. Voorhoeve, P. \& Agami, R. Tumor-suppressive functions of the human INK4A locus. Cancer Cell 4, 311-319 (2003).

15. Brummelkamp, T.R. \& Bernards, R. New tools for functional mammalian cancer genetics. Nat. Rev. Cancer 3, 781-789 (2003).

16. Brummelkamp, T.R. et al. An shRNA bar code screen provides insight into cancer cell vulnerability to MDM2 inhibitors. Nat. Chem. Biol. 2, 202-206 (2006).

17. Silva, J.M. et al. Second-generation shRNA libraries covering the mouse and human genomes. Nat. Genet. 37, 1281-1288 (2005).

18. Paddison, P.J. et al. A resource for large-scale RNA-interference-based screens in mammals. Nature 428, 427-431 (2004).

19. Brummelkamp, T.R. et al. Functional identification of cancer-relevant genes through large-scale RNA interference screens in mammalian cells. Cold Spring Harb. Symp. Quant. Biol. 69, 439-445 (2004).

20. Dirac, A.M., Nijman, S.M., Brummelkamp, T.R. \& Bernards, R. Functional annotation of deubiquitinating enzymes using RNA interference. Methods Enzymol. 398, 554-567 (2005).

21. Westbrook, T.F. et al. A genetic screen for candidate tumor suppressors identifies REST. Cell 121, 837-848 (2005).

22. Nijman, S.M. et al. The deubiquitinating enzyme USP1 regulates the Fanconi Anemia pathway. Mol. Cell 17, 331-339 (2005).

23. Kolfschoten, I.G. et al. A genetic screen identifies PITX1 as a suppressor of RAS activity and tumorigenicity. Cell 121, 849-858 (2005).

24. Hartwell, L.H., Szankasi, P., Roberts, C.J., Murray, A.W. \& Friend, S.H. Integrating genetic approaches into the discovery of anticancer drugs. Science 278, 1064-1068 (1997)

25. Moffat, J. et al. A lentiviral RNAi library for human and mouse genes applied to an arrayed viral high-content screen. Cell 124, 1283-1298 (2006).

26. Brummelkamp, T.R., Nijman, S.M., Dirac, A.M. \& Bernards, R. Loss of the cylindromatosis tumour suppressor inhibits apoptosis by activating NF- $\kappa B$. Nature 424, 797-801 (2003).

27. Nicke, B. et al. Involvement of MINK, a Ste20 family kinase, in Ras oncogeneinduced growth arrest in human ovarian surface epithelial cells. Mol. Cell 20, 673-685 (2005) 